\title{
Core-Valence Attosecond Transient Absorption Spectroscopy of Polyatomic Molecules
}

\author{
Nikolay V. Golubev@* and Jiří Vaníček \\ Laboratory of Theoretical Physical Chemistry, Institut des Sciences et Ingénierie Chimiques, \\ Ecole Polytechnique Fédérale de Lausanne (EPFL), CH-1015 Lausanne, Switzerland \\ Alexander I. Kuleff \\ Theoretische Chemie, Universität Heidelberg, Im Neuenheimer Feld 229, D-69120 Heidelberg, Germany \\ and ELI-ALPS, Wolfgang Sandner utca 3, H-6728 Szeged, Hungary
}

(Received 29 October 2020; revised 1 July 2021; accepted 30 July 2021; published 16 September 2021)

\begin{abstract}
Tracing ultrafast processes induced by interaction of light with matter is often very challenging. In molecular systems, the initially created electronic coherence becomes damped by the slow nuclear rearrangement on a femtosecond timescale which makes real-time observations of electron dynamics in molecules particularly difficult. In this work, we report an extension of the theory underlying the attosecond transient absorption spectroscopy (ATAS) for the case of molecules, including a full account for the coupled electron-nuclear dynamics in the initially created wave packet, and apply it to probe the oscillations of the positive charge created after outer-valence ionization of the propiolic acid molecule. By taking advantage of element-specific core-to-valence transitions induced by $\mathrm{x}$-ray radiation, we show that the resolution of ATAS makes it possible to trace the dynamics of electron density with atomic spatial resolution.
\end{abstract}

DOI: 10.1103/PhysRevLett.127.123001

The impressive progress in laser technologies during the last few decades $[1,2]$ has stimulated the rapid development of atomic and molecular physics. With the advent of ultrashort laser pulses, the scientific community obtained a unique tool to study such phenomena as charge transport in molecules [3,4] and the elementary steps of chemical reactions $[5,6]$, to name a few. Being able to observe fundamental processes with attosecond temporal resolution, it also becomes possible to steer and probe electron dynamics on its natural timescale [7].

One phenomenon of particular interest for the attosecond science is the process of ultrafast charge migration driven solely by the electron correlation and electron relaxation [8]. Charge migration arises whenever a coherent superposition of multiple electronic states is prepared, which, due to the electron correlation, can be achieved even when one removes an electron from a single molecular orbital. Such a coherent population of states can thus result from ionization of both outer- and inner-valence shells of a molecule [9-11], and even by removing an electron from localized core orbitals [12]. The dynamics triggered by the ionization manifests as a migration of the initially created localized charge through the system.

Published by the American Physical Society under the terms of the Creative Commons Attribution 4.0 International license. Further distribution of this work must maintain attribution to the author(s) and the published article's title, journal citation, and DOI.
Although much had been achieved in the theoretical understanding of the ultrafast electronic processes taking place in molecules $[13,14]$, the experimental demonstration of the electronic dynamics is still in its infancy [15]. So far, only a very limited number of experimental studies have been performed that were able to explore the correlated electron motion in complex systems. Evidence of ultrafast charge migration in amino acids phenylalanine $[16,17]$ and tryptophan [18] was demonstrated by measuring the yield of a doubly charged ion as a function of the delay between the ionizing pump and doubly ionizing probe pulses.

An alternative technique, using a rescattering of the ionized electron as a probe, is to measure the resulting highharmonic generation (HHG) spectra [19,20]. In the seminal work by Kraus et al. [21], attosecond charge migration in ionized iodoacetylene was reconstructed and controlled by analyzing the HHG spectrum emitted after irradiation of the molecule with strong infrared pulses of different wavelengths $(800$ and $1300 \mathrm{~nm}$ ). Despite being a promising experimental technique, the HHG spectroscopy can currently be used to capture only the first few femtoseconds of the dynamics thus preventing a simultaneous observation of the initial coherent electron motion and the follow-up effects caused by the coupling between the fast moving electrons and the slower nuclei.

Another experimental approach which combines high spectral and high temporal resolution is the attosecond transient absorption spectroscopy (ATAS) [2224]. Measuring the transmission of a broadband laser pulse 
through a sample, one can gain intuitive and highly detailed insights about dynamics of the system. In the pioneering work by Goulielmakis et al. [22], the ATAS was used to trace in real-time the valence electron motion in strongfield-generated $\mathrm{Kr}^{+}$ions. The straightforward interpretation of the ATAS [23] makes this technique a promising tool to study ultrafast dynamics in more complex molecular systems [25-29].

A particularly appealing feature of ATAS is the possibility to trace the evolution of the system with atomic spatial resolution by probing electron motion at a specific site of the molecule, as originally proposed by Dutoi et al. $[30,31]$. The latter is possible by taking advantage of element-specific core-to-valence transitions induced by $\mathrm{x}$-ray radiation. Recently, experimental setups providing soft $\mathrm{x}$-ray pulses with sub-femto-second duration were reported in both tabletop [32,33] and free electron laser facilities [34]. ATAS, therefore, appears as a very promising technique for the investigation of the charge migration dynamics triggered by ionization [35].

So far, the theory underlying ATAS has only been developed for atoms [23,36,37], or for molecules either in absence of nuclear motion [25,38] or in absence of electronic coherence [26]. At the same time, extensive $a b$ initio calculations for several molecules demonstrated [39-42] that coupling between electronic and nuclear degrees of freedom has a dramatic impact on the electron dynamics and thus has to be taken into account when computing the transient absorption spectrum. While the extensions of the ATAS formalism to the case of molecules $[43,44]$ have been widely used to support recent experimental measurements (see, e.g., Refs. [32,45]), the reported schemes are all based on the numerical analysis of various correlation functions which are difficult to interpret and numerically demanding to compute. In contrast, following the procedure reported in Ref. [23] for the case of atoms, we present a simple quasianalytical expression for the absorption cross section of molecules, which accounts for the nuclear motion and nonadiabatic dynamics and is composed from physically intuitive terms.

In this Letter, we extend the theory of ATAS to the case of polyatomic molecules, fully accounting for the coupled electron-nuclear dynamics in the initially created wave packet. We demonstrate on the example of propiolic acid that the resolution of ATAS is sufficient to clearly observe the ultrafast oscillations of the electron density, as well as the follow-up decoherence caused by the nuclear rearrangement. We perform high-level ab initio simulations of $\mathrm{x}$-ray ATAS, showing the possibility to trace the dynamics of the created hole through the molecular chain, and thus demonstrate that $\mathrm{x}$-ray ATAS can be a very useful technique to study ultrafast electron motion in molecules.

Ionization of a molecule by an ultrashort laser pulse brings the system to a nonstationary superposition of ionic states

$$
\Psi(\mathbf{r}, \mathbf{R}, t)=\sum_{I} \chi_{I}(\mathbf{R}, t) \Phi_{I}(\mathbf{r}, \mathbf{R}),
$$

where $\mathbf{r}$ and $\mathbf{R}$ denote electronic and nuclear coordinates, respectively, $\Phi_{I}(\mathbf{r}, \mathbf{R})$ are the electronic eigenstates obtained by solving the stationary Schrödinger equation $\hat{H}_{e} \Phi_{I}(\mathbf{r}, \mathbf{R})=E_{I}(\mathbf{R}) \Phi_{I}(\mathbf{r}, \mathbf{R})$ with electronic Hamiltonian $\hat{H}_{e}$, and $\chi_{I}(\mathbf{R}, t)$ are the expansion coefficients representing nuclear wave packets moving on the corresponding potential energy surfaces (PESs) $E_{I}(\mathbf{R})$. The ansatz [Eq. (1)] for the full molecular wave function is, in principle, an exact way to describe the concerted motion of electrons and nuclei in a molecule.

The electronic and nuclear dynamics induced after ionization of a system can be probed by analyzing the absorption of a spectrally broadband short laser pulse. Employing the time-dependent perturbation theory and Condon approximation, the absorption cross section can be calculated as

$$
\begin{aligned}
\sigma(\omega, \tau)= & \frac{4 \pi \omega}{c} \operatorname{Im} \sum_{I} \sum_{J}\left\langle\chi_{I}(\mathbf{R}, \tau) \mid \chi_{J}(\mathbf{R}, \tau)\right\rangle_{\mathbf{R}} \\
& \times \sum_{F}\left\langle\Phi_{I}|\hat{\mu}| \Phi_{F}\right\rangle\left\langle\Phi_{F}|\hat{\mu}| \Phi_{J}\right\rangle \\
& \times\left(\frac{1}{\tilde{E}_{F}-E_{I}-\omega}+\frac{1}{\tilde{E}_{F}^{*}-E_{J}+\omega}\right),
\end{aligned}
$$

where $\omega$ is the photon energy, $\tau$ is the delay between pump and probe pulses, $c$ is the speed of light in vacuum, $\left\langle\Phi_{I}|\hat{\mu}| \Phi_{F}\right\rangle$ and $\left\langle\Phi_{F}|\hat{\mu}| \Phi_{J}\right\rangle$ denote transition dipole matrix elements between initial $\{I, J\}$ and final $F$ electronic states with corresponding energies $E_{\{I, J, F\}}$ computed at fixed geometry $\mathbf{R}_{0}$, and quantities $\left\langle\chi_{I}(\mathbf{R}, \tau) \mid \chi_{J}(\mathbf{R}, \tau)\right\rangle_{\mathbf{R}}$ represent populations of initial electronic states when $I=J$ and the electronic coherences when $I \neq J$. To account for the broadening of the spectrum, we assign the final states a finite lifetime $1 / \Gamma$, which enters Eq. (2) in form of complex final state energies $\tilde{E}_{F}=E_{F}-i(\Gamma / 2)$. The detailed derivation of Eq. (2) is presented in Sec. I of the Supplemental Material [46].

As one can see from Eq. (2), the signal represents a combination of contributions corresponding to $\Lambda$-type transitions which couple initially populated $I$ and $J$ states through intermediate excited states $F$. The possibility to measure electron dynamics between states $I$ and $J$ is based on the fact that the interference between absorption of a photon with energy $\omega_{1}$ and stimulated emission of a photon with a different energy $\omega_{2}$ takes place only if the initial states are populated coherently. The electronic coherence term $\left\langle\chi_{I}(\mathbf{R}, \tau) \mid \chi_{J}(\mathbf{R}, \tau)\right\rangle_{\mathbf{R}}$ is the only source of time dependence in Eq. (2). Therefore, all the dynamical information required for calculations of absorption cross section comes from the propagation of nuclear wave packets $\chi_{I}(\mathbf{R}, t)$ in the initially populated electronic states 
while final states define energy positions and intensities of the corresponding lines in the spectrum.

Numerous theoretical approaches for computing electronic coherences were developed in the last few years [39-42,61]. Here, we use the recently described [42] semiclassical thawed Gaussian approximation (TGA) scheme [62-65], which combines efficient single trajectory evaluation of nuclear dynamics with fully ab initio on-thefly simulations of electronic structure. Within the TGA, the nuclear wave function is described by a single Gaussian wave packet whose center follows Hamilton's equations of motion and whose time-dependent width and phase are propagated using the local harmonic approximation of the PES. Despite its simplicity, the TGA gives results comparable in accuracy to the full dimensional quantum calculations when the involved states are not strongly coupled by nonadiabatic effects, as explicitly shown for propiolic acid [42].

To describe the electronic states of the ionized system, we use the high-level $a b$ initio algebraic diagrammatic construction (ADC) scheme [66] for representing the oneparticle Green's function. The ionization out of the valence orbitals is computed at the third-order ADC [ADC(3)] [67], while core ionic states are modeled using the fourth-order scheme [ADC(4)] [68]. Separate treatment of valence and core orbitals is dictated by the fact that the numerical efforts required for constructing and diagonalizing the ADC(4) secular matrix are considerable and prevent for the moment a straightforward application to the valence ionization of all but the smallest systems. At the same time, accurate description of core electrons requires an explicit account for the large relaxation effects while the correlation between the core and the valence electrons can be neglected. The ADC(4) scheme has been shown many times to yield highly accurate results when applied to core ionization [69]. Standard double-zeta plus polarization basis sets [70] were employed to construct the noncorrelated reference states. Ground-state geometry of the neutral molecule was optimized using the Gaussian 16 implementation [71] of the density functional theory [72] with the B3LYP functional [73].

Ionization spectrum of the propiolic acid is shown in Fig. 1. The first four valence ionic states are energetically separated by $2.8 \mathrm{eV}$ from the remaining ones. Due to the electron correlation, the ground and the second excited ionic states of the molecule are a strong mixture of two onehole configurations: an electron missing in the highest occupied molecular orbital (HOMO) and an electron missing in the HOMO-2. Therefore, a sudden removal of an electron either from HOMO or from HOMO-2 will create an electronic wave packet, which will initiate charge migration oscillations between the carbon triple bond and the carbonyl oxygen with a period of about $4.5 \mathrm{fs}$, determined by the energy gap between the first and the third cationic states $[41,74]$. The two orbitals involved in
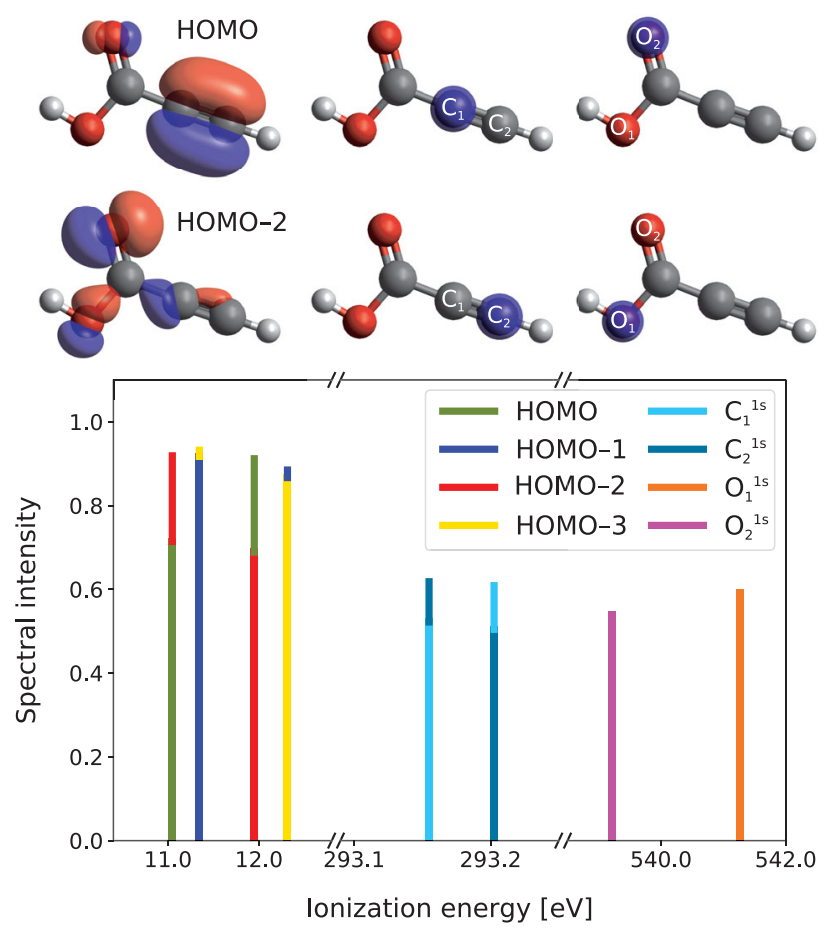

FIG. 1. Ionization spectrum of propiolic acid computed using the $a b$ initio many-body Green's function ADC method. Three energy windows illustrate the valence region $(10.5-12.5 \mathrm{eV})$, core ionic states resulting from ionization out of $1 s$ orbitals of triple-bond carbon atoms (293.1-293.25 eV), and oxygen atoms (539-542 eV). In all three energy ranges, the closest ionic states are located at least $\sim 2.5 \mathrm{eV}$ above the presented lines. The spectral intensity is defined as the combined weight of all onehole configurations in the configuration-interaction expansion of the ionic state. The relevant molecular orbitals are also depicted.

this hole mixing are also depicted in Fig. 1. Importantly, the molecule has planar symmetry and thus belongs to the $C_{s}$ symmetry group which allows assignment of the ionic states to two irreducible representations: the first and third states belong to the $A^{\prime}$, while the second and fourth states to $A^{\prime \prime}$. The latter allows one to obtain the desired superposition of the first and third ionic states by appropriately orienting the molecule with respect to the laser polarization.

Let us now look at the states corresponding to ionization out of the core orbitals of the molecule. Due to the fact that $1 s$ core orbitals are localized in space, it is possible to associate every core orbital with a specific atom in the system. Figure 1 depicts ionic states resulting from ionization out of core orbitals of oxygen and carbon atoms of the propiolic acid. The spectrum is plotted for two energy windows which capture ionic states with dominant contributions of the corresponding core orbitals. Interestingly, core orbitals of carbon atoms forming the triple-bond experience strong hole mixing similar to the one reported previously for ionization out of valence and upper-range inner orbitals only $[9,13]$. We would like to note in passing that due to the small energy difference between these core 
ionic states, the electronic oscillations for the wave packet resulting from such a mixture is expected to be significantly slower than those taking place in the case of valence ionization of the molecule. The ionic state resulting from ionization out of the $1 \mathrm{~s}$ orbital of a carbon atom of the carboxyl group is located $3.9 \mathrm{eV}$ above the states belonging to ionization out of orbitals of carbons forming the triple bond and is not shown in Fig. 1. Similarly, the closest satellite ionic states corresponding to ionization out of oxygen core orbitals are located $2.5 \mathrm{eV}$ above main lines. These energy gaps make it possible to distinguish energetically the transitions between valence ionic states and only those core states shown in Fig. 1 while neglecting transitions to other electronic states which are not covered by the presented energy ranges.

To account for transitions between initial valence $\left|\Phi_{\{I J\}}\right\rangle$ and final core $\left|\Phi_{F}\right\rangle$ states present in Eq. (2), one needs to evaluate the corresponding transition dipole matrix elements $\left\langle\Phi_{I}|\hat{\mu}| \Phi_{F}\right\rangle$ and $\left\langle\Phi_{F}|\hat{\mu}| \Phi_{J}\right\rangle$. The latter cannot be done directly, because our initial and final states are computed at different levels of electronic structure theory. An alternative way to perform these calculations is to exploit the configuration-interaction-like structure of the electronic wave functions appearing in the ADC approach. Details of this procedure can be found in Sec. II of the Supplemental Material [46] (see also Ref. [75]). Importantly, accurate values of the dipole transitions play a central role in the possibility to resolve spatial localization of the charge in a molecule. Due to the interference between photons connecting various combinations of initial $\{I, J\}$ and final $F$ states, scaled by the corresponding dipole transitions, one can infer a direct correspondence between the real-space dynamics taking place in a system under study and the ATAS signal computed via Eq. (2).

We performed calculations of ATAS for the evolution of the wave packet created by projecting the ground (electronic and nuclear) neutral state of the propiolic acid onto the first and third cationic states of the molecule employing the sudden and Franck-Condon approximations. The initial phase between involved electronic states is chosen in such a way that the created charge is localized in the HOMO. The top panel of Fig. 2 shows the evolution of the electronic coherence between initially populated states, i.e., the matrix element $\left\langle\chi_{1}(\mathbf{R}, \tau) \mid \chi_{3}(\mathbf{R}, \tau)\right\rangle_{\mathbf{R}}$, in time. It is seen that the electronic oscillations are strongly influenced by the nuclear motion and the coherence is completely suppressed within the first 15 fs $[41,42]$. The middle and bottom panels of Fig. 2 depict the absorption cross section computed using Eq. (2) for transitions between initially populated valence ionic states and the core states, resulting from ionization out of $1 s$ orbitals of carbon and oxygen atoms, respectively. Line positions in ATAS are determined by the energy gaps between the initial and final electronic states. Transitions from the two initially populated valence states to the two final oxygen core ionic states lead to the

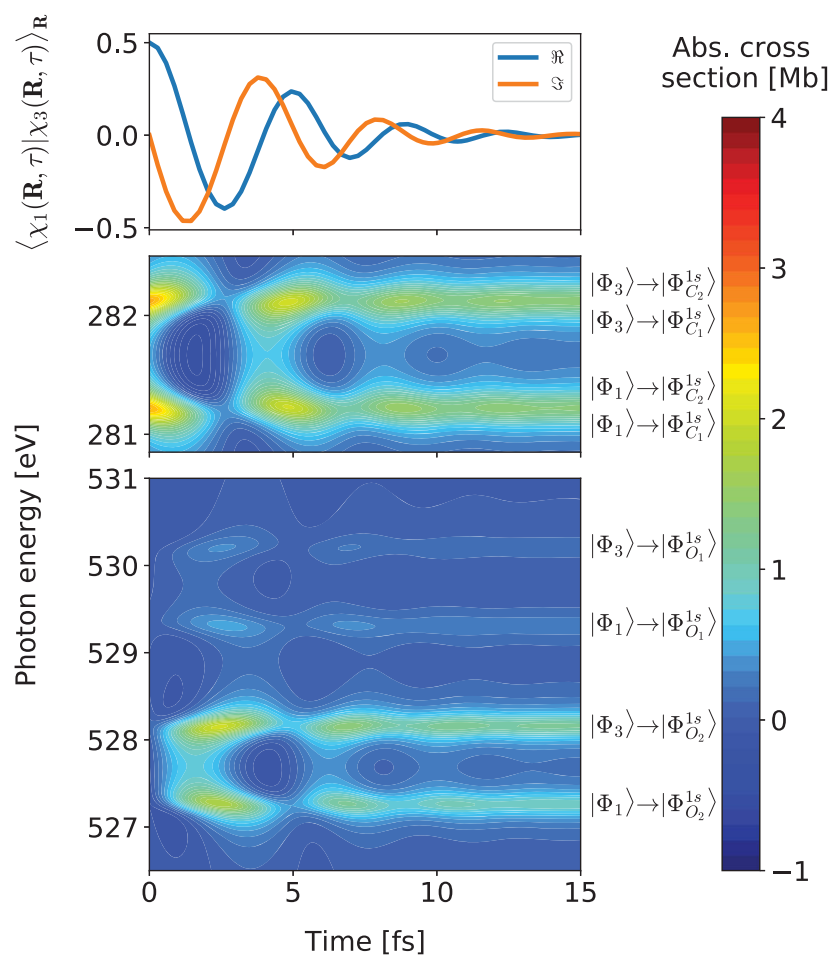

FIG. 2. Top: electronic coherence measured by the timedependent overlap $\left\langle\chi_{1}(\mathbf{R}, \tau) \mid \chi_{3}(\mathbf{R}, \tau)\right\rangle_{\mathbf{R}}$ of the nuclear wave packets propagated in the first and third cationic states of propiolic acid after the removal of an electron from the HOMO. Middle: time-resolved absorption cross section as a function of the photon energy and time delay, plotted for the energy window corresponding to transitions between initially populated valence ionic states and the core states resulting from ionization out of $1 s$ orbitals of carbon atoms forming the triple bond. Bottom: absorption cross section plotted for the energy range covering transitions to core ionic states of the oxygen atoms. The ionic states involved in the corresponding transitions are also shown. The transient absorption traces shown are rotationally averaged and thus describe randomly oriented molecules.

appearance of four lines in the spectrum. In the case of carbon core ionic states, the four absorption lines appear as two visible transitions due to the small energy gap between the final core states. The intensity of the lines is determined by the product of the transition dipole moments that couple initial and final states via $\Lambda$-type transitions. All calculations were rotationally averaged and thus describe randomly oriented molecules (see Sec. III of the Supplemental Material [46]). An energy broadening parameter $\Gamma=0.3 \mathrm{eV}$ was used (see Sec. I of the Supplemental Material [46]).

Spectra in Fig. 2 clearly illustrate that the resolution of ATAS makes it possible to trace the real-time oscillatory charge migration dynamics before the nuclear motion dephases the electron coherence. Importantly, the maxima in the beatings of the absorption lines corresponding to core excitations of both carbon and oxygen atoms correlate with the spatial localization of the hole density in the vicinity of 
these atoms. The latter allows us to trace the electron dynamics along the molecular chain with atomic spatial resolution. It is seen that immediately after the ionization the absorption takes place almost exclusively on the triplebond carbon atoms suggesting that the initial hole charge is located in the triple bond of the molecule. Within $2 \mathrm{fs}$, the charge density migrates to the carboxyl group of the molecule which is reflected by the strong absorption signal from the oxygen atoms, mainly from the carbonyl oxygen. After several oscillations, the lines become stationary yet remain present for all shown core states, which reflects the fact that the charge distributes almost uniformly along the molecular chain.

Before concluding, we emphasize that Eq. (2) is obtained with the assumption that the initial time-dependent state of the system, Eq. (1), is prepared before the action of the probe pulse. In other words, we utilize the first-order polarization response of the system with respect to the applied electric field, operating in a regime of nonoverlapping pump and probe pulses. Although being a limitation, this treatment of the light-matter interaction allows us to simulate ATAS with the time-dependent perturbation theory applied to a nonstationary initial state of a system that is, in turn, described in a completely nonperturbative fashion. Another major approximation used in this study is the short-pulse approximation for the probe pulse. The latter requirement means that the duration of the probe pulse should be shorter than the oscillations of the coherences in the initially prepared superposition. The reported scheme suggests simultaneous measurements of the absorption cross section for both carbon and oxygen core ionic states of the propiolic acid which automatically makes the required laser pulse short enough to cover this broad energy range. An ideal experiment would involve ultrashort extreme-ultraviolet-pump and x-rayprobe pulses, although the ionization step can be also performed via other mechanisms, e.g., strong-field multiphoton absorption. In the latter case, the initially created superposition can be sensitive to the variations of the laser pulse parameters which will lead to the loss of the coherence and thus to less pronounced ATAS signal (see Sec. IV of the Supplemental Material [46]). Nevertheless, as was already pointed out in the introduction, modern experimental setups [33,34] provide radiation with the unique combination of high intensity, high photon energy, and short pulse duration which can open the door for realtime studies of the electron dynamics in complex molecules using ATAS technique. However, because the electronic states belonging to the ionization out of core orbitals of the same atoms lie typically very close to each other in energy (see, e.g., carbon ionic states in Fig. 1), the resolution of ATAS might be limited to trace the dynamics only between atoms of different chemical elements, or between differently bonded same elements giving rise to larger chemical shifts.
In conclusion, we have demonstrated the application of ATAS to probe the ultrafast dynamics of electron density in a polyatomic molecule. We presented the extension of the theory underlying ATAS taking into account coupling between electronic and nuclear degrees of freedom. We performed high-level ab initio calculations of ATAS for the propiolic acid demonstrating the possibility to detect both temporal and spatial aspects of the electron dynamics. Our findings illustrate that the resolution of ATAS in the soft $\mathrm{x}$-ray energy range is sufficient to identify structural groups and even particular atoms involved in the process. Finally, we observed strong hole mixing between core orbitals forming the triple bond of a molecule. The investigation of possible dynamics resulting from ionization out of core orbitals is a promising direction of further research. We hope that our work will motivate such studies.

N. V. G. acknowledges the support by the Branco Weiss Fellowship-Society in Science, administered by the ETH Zürich. J. V. acknowledges the Swiss National Science Foundation for financial support through the National Center of Competence in Research MUST (Molecular Ultrafast Science and Technology) Network. A. I. K. is thankful for the financial support provided by the DFG through the QUTIF Priority Programme and the US ARO (Grant No. W911NF-14-1-0383).

*nik.v.golubev@gmail.com

[1] F. Krausz and M. Ivanov, Rev. Mod. Phys. 81, 163 (2009).

[2] F. Calegari, G. Sansone, S. Stagira, C. Vozzi, and M. Nisoli, J. Phys. B 49, 062001 (2016).

[3] K. L. M. Lewis and J. P. Ogilvie, J. Phys. Chem. Lett. 3, 503 (2012).

[4] H. J. Wörner, C. A. Arrell, N. Banerji, A. Cannizzo, M. Chergui, A. K. Das, P. Hamm, U. Keller, P. M. Kraus, E. Liberatore, P. Lopez-Tarifa, M. Lucchini, M. Meuwly, C. Milne, J.-E. Moser, U. Rothlisberger, G. Smolentsev, J. Teuscher, J. A. van Bokhoven, and O. Wenger, Struct. Dyn. 4, 061508 (2017).

[5] A. H. Zewail, J. Phys. Chem. A 104, 5660 (2000).

[6] S. Baker, J. S. Robinson, C. A. Haworth, H. Teng, R. A. Smith, C. C. Chirila, M. Lein, J. W. G. Tisch, and J.P. Marangos, Science 312, 424 (2006).

[7] K. Ramasesha, S. R. Leone, and D. M. Neumark, Annu. Rev. Phys. Chem. 67, 41 (2016).

[8] L. Cederbaum and J. Zobeley, Chem. Phys. Lett. 307, 205 (1999)

[9] J. Breidbach and L. S. Cederbaum, J. Chem. Phys. 118, 3983 (2003).

[10] S. Lünnemann, A. I. Kuleff, and L. S. Cederbaum, J. Chem. Phys. 129, 104305 (2008)

[11] S. Lünnemann, A. I. Kuleff, and L. S. Cederbaum, J. Chem. Phys. 130, 154305 (2009).

[12] A. I. Kuleff, N. V. Kryzhevoi, M. Pernpointner, and L. S. Cederbaum, Phys. Rev. Lett. 117, 093002 (2016).

[13] A. I. Kuleff and L. S. Cederbaum, J. Phys. B 47, 124002 (2014). 
[14] M. Nisoli, P. Decleva, F. Calegari, A. Palacios, and F. Martín, Chem. Rev. 117, 10760 (2017).

[15] S. R. Leone, C. W. McCurdy, J. Burgdörfer, L. S. Cederbaum, Z. Chang, N. Dudovich, J. Feist, C. H. Greene, M. Ivanov, R. Kienberger, U. Keller, M. F. Kling, Z.-H. Loh, T. Pfeifer, A. N. Pfeiffer, R. Santra, K. Schafer, A. Stolow, U. Thumm, and M. J. J. Vrakking, Nat. Photonics 8, 162 (2014).

[16] L. Belshaw, F. Calegari, M. J. Duffy, A. Trabattoni, L. Poletto, M. Nisoli, and J. B. Greenwood, J. Phys. Chem. Lett. 3, 3751 (2012).

[17] F. Calegari, D. Ayuso, A. Trabattoni, L. Belshaw, S. De Camillis, S. Anumula, F. Frassetto, L. Poletto, A. Palacios, P. Decleva, J. B. Greenwood, F. Martin, and M. Nisoli, Science 346, 336 (2014).

[18] M. Lara-Astiaso, M. Galli, A. Trabattoni, A. Palacios, D. Ayuso, F. Frassetto, L. Poletto, S. De Camillis, J. Greenwood, P. Decleva, I. Tavernelli, F. Calegari, M. Nisoli, and F. Martín, J. Phys. Chem. Lett. 9, 4570 (2018).

[19] O. Smirnova, Y. Mairesse, S. Patchkovskii, N. Dudovich, D. Villeneuve, P. Corkum, and M. Y. Ivanov, Nature (London) 460, 972 (2009).

[20] Y. Mairesse, J. Higuet, N. Dudovich, D. Shafir, B. Fabre, E. Mével, E. Constant, S. Patchkovskii, Z. Walters, M. Y. Ivanov, and O. Smirnova, Phys. Rev. Lett. 104, 213601 (2010).

[21] P. M. Kraus, B. Mignolet, D. Baykusheva, A. Rupenyan, L. Horny, E. F. Penka, G. Grassi, O. I. Tolstikhin, J. Schneider, F. Jensen, L. B. Madsen, A. D. Bandrauk, F. Remacle, and H. J. Wörner, Science 350, 790 (2015).

[22] E. Goulielmakis, Z.-H. Loh, A. Wirth, R. Santra, N. Rohringer, V.S. Yakovlev, S. Zherebtsov, T. Pfeifer, A. M. Azzeer, M. F. Kling, S. R. Leone, and F. Krausz, Nature (London) 466, 739 (2010).

[23] R. Santra, V. S. Yakovlev, T. Pfeifer, and Z.-H. Loh, Phys. Rev. A 83, 033405 (2011).

[24] A. Wirth, R. Santra, and E. Goulielmakis, Chem. Phys. 414, 149 (2013).

[25] L. Drescher, G. Reitsma, T. Witting, S. Patchkovskii, J. Mikosch, and M. J. J. Vrakking, J. Phys. Chem. Lett. 10, 265 (2019).

[26] Y. Kobayashi, K. F. Chang, T. Zeng, D. M. Neumark, and S. R. Leone, Science 365, 79 (2019).

[27] Y. Kobayashi, D. M. Neumark, and S. R. Leone, Phys. Rev. A 102, 051102 (2020).

[28] Y. Kobayashi, K. F. Chang, S. M. Poullain, V. Scutelnic, T. Zeng, D. M. Neumark, and S. R. Leone, Phys. Rev. A 101, 063414 (2020).

[29] H. Timmers, X. Zhu, Z. Li, Y. Kobayashi, M. Sabbar, M. Hollstein, M. Reduzzi, T. J. Martínez, D. M. Neumark, and S. R. Leone, Nat. Commun. 10, 3133 (2019).

[30] A. D. Dutoi, K. Gokhberg, and L. S. Cederbaum, Phys. Rev. A 88, 013419 (2013).

[31] A. D. Dutoi and L. S. Cederbaum, Phys. Rev. A 90, 023414 (2014).

[32] N. Saito, H. Sannohe, N. Ishii, T. Kanai, N. Kosugi, Y. Wu, A. Chew, S. Han, Z. Chang, and J. Itatani, Optica 6, 1542 (2019).

[33] L. Barreau, A. D. Ross, S. Garg, P. M. Kraus, D. M. Neumark, and S. R. Leone, Sci. Rep. 10, 5773 (2020).
[34] J. Duris et al., Nat. Photonics 14, 30 (2020).

[35] J. Li, J. Lu, A. Chew, S. Han, J. Li, Y. Wu, H. Wang, S. Ghimire, and Z. Chang, Nat. Commun. 11, 2748 (2020).

[36] S. Pabst, A. Sytcheva, A. Moulet, A. Wirth, E. Goulielmakis, and R. Santra, Phys. Rev. A 86, 063411 (2012).

[37] D. Kolbasova and R. Santra, Appl. Sci. 9, 1350 (2019).

[38] M. Hollstein, R. Santra, and D. Pfannkuche, Phys. Rev. A 95, 053411 (2017).

[39] C. Arnold, O. Vendrell, and R. Santra, Phys. Rev. A 95, 033425 (2017).

[40] M. Vacher, M. J. Bearpark, M. A. Robb, and J. P. Malhado, Phys. Rev. Lett. 118, 083001 (2017).

[41] V. Despré, N. V. Golubev, and A. I. Kuleff, Phys. Rev. Lett. 121, 203002 (2018).

[42] N. V. Golubev, T. Begušić, and J. Vaníček, Phys. Rev. Lett. 125, 083001 (2020).

[43] J. E. Bækhøj and L. B. Madsen, Phys. Rev. A 94, 043414 (2016).

[44] J. J. Rørstad, N. S. W. Ravn, L. Yue, and L. B. Madsen, Phys. Rev. A 98, 053401 (2018).

[45] C.-T. Liao, X. Li, D. J. Haxton, T. N. Rescigno, R. R. Lucchese, C. W. McCurdy, and A. Sandhu, Phys. Rev. A 95, 043427 (2017).

[46] See Supplemental Material at http://link.aps.org/ supplemental/10.1103/PhysRevLett.127.123001 for the derivation of the expression for computing the absorption cross section, description of the approach for evaluating transition properties between electronic states, and also the averaging schemes, which includes Refs. [47-60].

[47] D. J. Tannor, Introduction to Quantum Mechanics: A Time-Dependent Perspective (University Science Books, Sausalito, 2007).

[48] A. I. Kuleff and L. S. Cederbaum, Phys. Rev. Lett. 106, 053001 (2011).

[49] S. Mukamel, Principles of Nonlinear Optical Spectroscopy, Oxford Series in Optical and Imaging Sciences No. 6 (Oxford University Press, New York, 1995).

[50] R. Sankari, M. Ehara, H. Nakatsuji, Y. Senba, K. Hosokawa, H. Yoshida, A. De Fanis, Y. Tamenori, S. Aksela, and K. Ueda, Chem. Phys. Lett. 380, 647 (2003).

[51] T. X. Carroll, J. Hahne, T. D. Thomas, L. J. Sæthre, N. Berrah, J. Bozek, and E. Kukk, Phys. Rev. A 61, 042503 (2000).

[52] R. Geneaux, H. J. B. Marroux, A. Guggenmos, D. M. Neumark, and S. R. Leone, Phil. Trans. R. Soc. A 377, 20170463 (2019).

[53] T. Helgaker, P. Jørgensen, and J. Olsen, Molecular Electronic-Structure Theory (Wiley, New York, 2000).

[54] A. I. Krylov, Annu. Rev. Phys. Chem. 59, 433 (2008).

[55] J. Schirmer, Many-Body Methods for Atoms, Molecules and Clusters, Lecture Notes in Chemistry Vol. 94 (Springer International Publishing, Cham, 2018).

[56] J. C. Slater, Phys. Rev. 34, 1293 (1929).

[57] E. U. Condon, Phys. Rev. 36, 1121 (1930).

[58] J. Schirmer and A. B. Trofimov, J. Chem. Phys. 120, 11449 (2004).

[59] A. B. Trofimov and J. Schirmer, J. Chem. Phys. 123, 144115 (2005). 
[60] T. Begušić, A. Patoz, M. Šulc, and J. Vaníček, Chem. Phys. 515, 152 (2018).

[61] M. Vacher, L. Steinberg, A. J. Jenkins, M. J. Bearpark, and M. A. Robb, Phys. Rev. A 92, 040502(R) (2015).

[62] E. J. Heller, J. Chem. Phys. 62, 1544 (1975).

[63] T. Begušić, J. Roulet, and J. Vaníček, J. Chem. Phys. 149, 244115 (2018).

[64] T. Begušić, M. Cordova, and J. Vaníček, J. Chem. Phys. 150, 154117 (2019).

[65] C. Lasser and C. Lubich, Acta Numer. 29, 229 (2020).

[66] J. Schirmer, L. S. Cederbaum, and O. Walter, Phys. Rev. A 28, 1237 (1983).

[67] J. Schirmer, A. B. Trofimov, and G. Stelter, J. Chem. Phys. 109, 4734 (1998).

[68] G. Angonoa, O. Walter, and J. Schirmer, J. Chem. Phys. 87, 6789 (1987).
[69] V. Feyer, O. Plekan, R. Richter, M. Coreno, G. Vall-llosera, K. C. Prince, A. B. Trofimov, I. L. Zaytseva, T. E. Moskovskaya, E. V. Gromov, and J. Schirmer, J. Phys. Chem. A 113, 5736 (2009).

[70] A. Canal Neto, E. Muniz, R. Centoducatte, and F. Jorge, J. Mol. Struct. 718, 219 (2005).

[71] M. J. Frisch et al., Gaussian 16 Revision C.01, (2016), gaussian Inc. Wallingford CT.

[72] P. Hohenberg and W. Kohn, Phys. Rev. 136, B864 (1964).

[73] P. J. Stephens, F. J. Devlin, C. F. Chabalowski, and M. J. Frisch, J. Phys. Chem. 98, 11623 (1994).

[74] N. V. Golubev, V. Despré, and A. I. Kuleff, J. Mod. Opt. 64, 1031 (2017).

[75] V. Pohl, G. Hermann, and J. C. Tremblay, J. Comput. Chem. 38, 1515 (2017). 